Proceedings

\title{
Detection of Ethanol in Human Breath Using Optical Fiber Long Period Grating Coated with Metal-Organic Frameworks ${ }^{\dagger}$
}

\author{
Nerea De Acha 1,*, Jiri Hromadka ${ }^{2}$, Begum Tokay ${ }^{3}$, Ricardo Correia ${ }^{2}$, Cesar Elosua ${ }^{1,4}$, \\ Ignacio R. Matías 1,4, Francisco J. Arregui 1,4, Stephen P. Morgan ${ }^{2}$ and Sergiy Korposh ${ }^{2}$ \\ 1 Department of Electrical and Electronic Engineering, Public University of Navarra, E-31006 Pamplona, \\ Spain; cesar.elosua@unavarra.es (C.E.); natxo@unavarra.es (I.R.M.); parregui@unavarra.es (F.J.A.) \\ 2 Applied Optics Group, Electrical Systems and Optics Research Division, Faculty of Engineering, \\ University of Nottingham, University Park, Nottingham NG7 2RD, UK; \\ jiri.hromadka@nottingham.ac.uk (J.H.); Ricardo.GoncalvesCorreia@nottingham.ac.uk (R.C.); \\ steve.morgan@nottingham.ac.uk (S.P.M.); S.Korposh@nottingham.ac.uk (S.K.) \\ 3 Department of Chemical and Environmental Engineering, University of Nottingham, University Park, \\ Nottingham NG7 2RD, UK; Begum.Tokay@nottingham.ac.uk \\ 4 Institute of Smart Cities, Public University of Navarra, E-31006 Pamplona, Spain \\ * Correspondence: nerea.deacha@unavarra.es; Tel.: +34-948-166-044 \\ + Presented at the Eurosensors 2017 Conference, Paris, France, 3-6 September 2017.
}

Published: 21 August 2017

\begin{abstract}
An optical fiber sensor for ethanol detection in exhaled breath has been developed. It has been fabricated by functionalizing a Long Period Grating with a metal-organic framework, ZIF-8. The sensor's response was tested by exposure to exhaled breath of a person before and after the ingestion of alcoholic drinks, showing a higher wavelength difference between the resonance bands in the second case. Further work will analyze cross-sensitivity towards temperature, relative humidity and carbon dioxide.
\end{abstract}

Keywords: ethanol detection; exhaled breath; optical fiber sensor; Long Period Grating; Metal-Organic Framework

\section{Introduction}

In recent years, there has been an increasing interest in monitoring Volatile Organic Compounds (VOCs) in exhaled breath [1], since they can be used as indicators of different illnesses [2,3]; in the specific case of ethanol, its measurement in breath is at high interest in traffic safety [4].

The most commonly employed technique for the quantitative analysis of these compounds is gas chromatography-mass spectroscopy (GC-MS) [5], which is expensive, time consuming and requires high experienced personnel. A wide variety of electronic sensors, most of them based on semiconductor technology is under development for VOCs detection [6], however, they present many drawbacks, such as their high response time [7], low selectivity, non-immunity to electromagnetic interferences [8] or the need of biasing [9]. Thus, there is a need for the development of more accurate, less expensive and short response time sensors. These requirements can be met by employing optical fiber as the sensing platform: it presents many advantages, such as the possibility of multiplexing more sensors using one interrogation unit, being used in hazardous environments [10] or its immunity towards electromagnetic interferences [11].

Long Period Gratings (LPG) are devices based on periodic perturbations of the refractive index of the fiber core that promote the coupling of the core mode to the co-propagating cladding ones [12]. This coupling can be observed in the transmission spectrum as a series a loss bands, whose central 
wavelength is sensitive to external conditions, such as temperature, bending or refractive index. In order to develop a VOC sensor based on LPG, this structure must be functionalized with a coating that is sensitive to the target VOC [13]. In this work, the LPG has been coated with a ZIF-8 sensing coating, which is a metal-organic framework that exhibits cavities for gas storage [14].

\section{Materials and Methods}

\subsection{Sensing Material}

Zeolitic Imidazolate Framework (ZIF-8) was chosen as sensing material. The structure of this metal-organic framework is made by zinc ions coordinated by four imidazolate rings, exhibiting large cavities that allow gas molecules storage [14]. It is also well-known due to its chemical robustness and its thermal stability [15] and it has been previously demonstrated that it can be used as sensitive material towards organic vapors [16].

\subsection{Long Period Grating}

A Long Period Grating consists of a periodic modification of the refractive index of the fiber core that promotes the coupling of the core mode to the co-propagating cladding modes [12]. Because of this coupling, different resonance bands can be observed in the transmission spectrum, whose central wavelength can be obtained by equation:

$$
\lambda_{X}=\left(n_{\text {core }}-n_{\text {clad }(X)}\right) \Delta
$$

where $\lambda_{X}$ is the wavelength at which light is coupled to the mode LP0X, $n_{\text {core }}$ is the effective refractive index of the core mode, $n_{\operatorname{clad}(X)}$ is the effective refractive index of the LPOX cladding mode and $\Delta$ is the period of the LPG.

\subsection{Sensor Construction Process}

An LPG with grating period of $109 \mu \mathrm{m}$ and length of $30 \mathrm{~mm}$ was fabricated onto boron-germanium co-doped optical fiber (Fibercore PS750) with a cut-off wavelength $670 \mathrm{~nm}$, using custom made amplitude mask. The optical fiber was side-illuminated by the output from a frequency-quadrupled Nd:YAG laser operating at $266 \mathrm{~nm}$ [17].

ZIF-8 was deposited onto the LPG by means of the in situ crystallization technique: the LPG was immersed for $30 \mathrm{~min}$ in a mixture of $15 \mathrm{~mL}$ of $2.5 \mathrm{mM}$ zinc nitrate hexahydrate and $15 \mathrm{~mL}$ of $25 \mathrm{mM}$ 2-metyl-imidazole. After that, it was washed with methanol and exposed to a nitrogen flow in order to be dried [18]. This process was repeated five times.

\subsection{Sensor Characterization}

Sensor's performance was analyzed by exposing it to the exhaled breath of a person before and after the ingestion of alcoholic drinks. Transmission spectra of the LPG were recorded every $500 \mathrm{~ms}$, and the central wavelengths of their resonance bands were monitored to evaluate the effect of the presence of ethanol in human breath. Temperature was also recorded using a thermocouple from PicoTechnology.

\section{Results and Discussion}

\subsection{Sensor Fabrication}

The changes in the transmission spectrum during the ZIF-8 in-situ crystallization on the surface of the LPG were monitored: results are displayed in Figure 1. The transmission spectra show the shift of the resonance bands corresponding to LP018 and LP019 cladding modes after the deposition of each growth cycle of ZIF-8, when the LPG was still immersed in the solution. The central wavelengths difference between the LP019 resonance bands linearly increases as the number of growth cycles increases, indicating the uniform deposition of ZIF-8 thin film. 


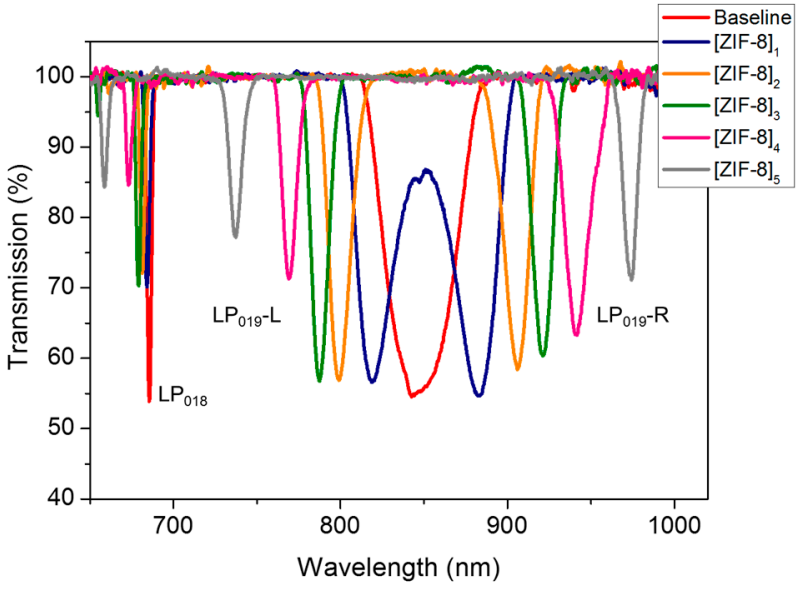

Figure 1. Transmission spectra of each growth cycle during immersion of the LPG in ZIF-8.

\subsection{Sensor Characterization}

The sensor's performance to detect ethanol in exhaled breath was analyzed by exposure to human breath before and after consuming alcoholic drinks. As it can be observed in Figure 2a, exhaled breath induced an increase on the spectral distance between resonance bands due the changes in temperature and relative humidity, which was further increased by the presence of ethanol, demonstrating the capability of the sensor to detect the presence of ethanol in exhaled breath.

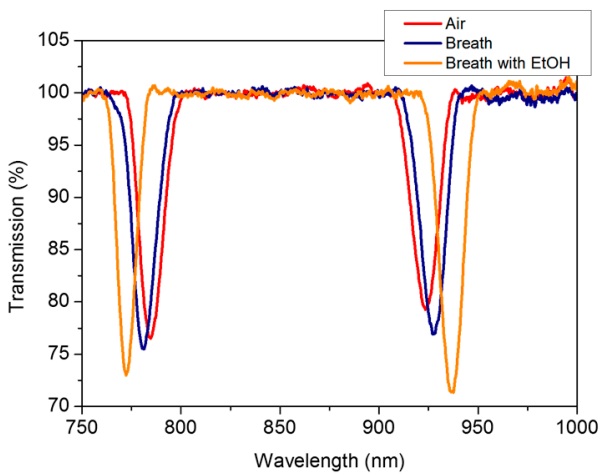

(a)

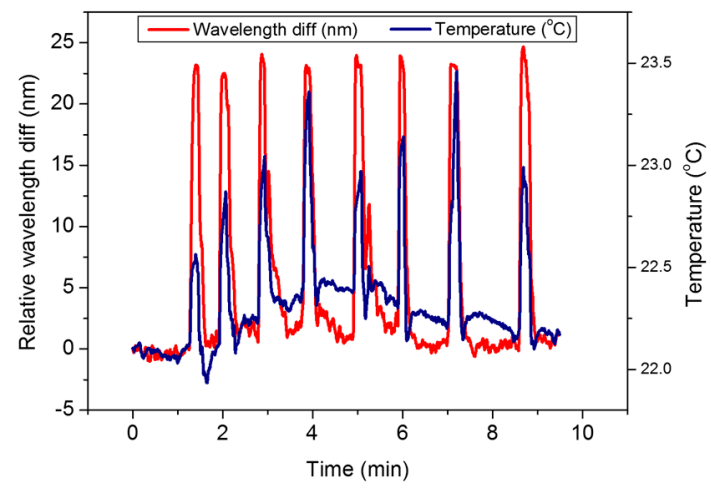

(b)

Figure 2. (a) Transmission spectra of the [ZIF-8]5 coated LPG in air (reference, red line) and when exposed to exhaled before (blue line) and after the ingestion of alcoholic drinks (yellow line); (b) Wavelength difference of the resonance bands when the sensor is exposed to exhaled breath after the ingestion of alcoholic drinks (red line) and temperature variation due to exhaled breath (blue line).

\section{Conclusions}

A sensor fabricated by depositing five growth cycles of metal-organic framework ZIF-8 onto a LPG with grating period of $109 \mu \mathrm{m}$ and length of $30 \mathrm{~mm}$ has been demonstrated to detect ethanol in exhaled breath. Wavelength separation between the resonance bands is higher when ethanol is present in exhaled breath than in its absence, independently on temperature. Further work will also analyze the effect of other parameters related to exhaled breath that could affect the performance of the sensor, such as relative humidity or the presence of other gases, for instance, carbon dioxide.

Acknowledgments: The authors would like to acknowledge Spanish Ministry of Economy and Competitiveness for the financial support through TEC2016-78047-R project.

Conflicts of Interest: The authors declare no conflict of interest. The founding sponsors had no role in the design of the study; in the collection, analyses, or interpretation of data; in the writing of the manuscript, and in the decision to publish the results. 


\section{References}

1. Wang, C.; Sahay, P. Breath analysis using laser spectroscopic techniques: Breath biomarkers, spectral fingerprints, and detection limits. Sensors 2009, 9, 8230-8262.

2. Ashutosh, K. Nitric oxide and asthma: A review. Curr. Opin. Pulm. Med. 2000, 6, 21-25,.

3. Paredi, P.; Shah, P.L.; Montuschi, P.; Sullivan, P.; Hodson, M.E.; Kharitonov, S.A.; Barnes, P.J. Increased carbon monoxide in exhaled air of patients with cystic fibrosis. Thorax 1999, 54, 917-920.

4. Guillem, D.-G.; Jordi, S.; Paolo, P.; Sven, B.; Lsabel, G.; Carles, C.; Albert, R.-R. Gas nanosensors based on individual indium oxide nanostructures. Procedia Eng. 2015, 120, 795-798.

5. Santos, F.J.; Galceran, M.T. Modern developments in gas chromatography-mass spectrometry-based environmental analysis. J. Chromatogr. A 2003, 1000, 125-151.

6. Elmi, I.; Zampolli, S.; Cozzani, E.; Mancarella, F.; Cardinali, G.C. Development of ultra-low-power consumption MOX sensors with ppb-level VOC detection capabilities for emerging applications. Sens. Actuators B Chem. 2008, 135, 342-351.

7. Szczurek, A.; Badura, M.; Banaszkiewicz, K.; Maciejewska, M.; Marcinkowski, T. Monitoring Volatile Organic Compound Emission Based on Semiconductor Gas Sensors. Environ. Eng. Sci. 2014, 31, 533-540.

8. Bohnert, K.; Gabus, P.; Kostovic, J.; Brändle, H. Optical fiber sensors for the electric power industry. Opt. Lasers Eng. 2005, 43, 511-526.

9. Meng, E.; Li, P.-Y.; Tai, Y.-C. A biocompatible Parylene thermal flow sensing array. Sens. Actuators A Phys. 2008, 144, 18-28.

10. Liu, T.; Wei, Y.; Song, G.; Li, Y.; Wang, J.; Ning, Y.; Lu, Y. Advances of optical fiber sensors for coal mine safety monitoring applications. In Proceedings of the International Conference on Microwave and Photonics (ICMAP), Dhanbad, India, 13-15 December 2013.

11. Lee, B. Review of the present status of optical fiber sensors. Opt. Fiber Technol. 2003, 9, 57-79.

12. James, S.W.; Tatam, R.P. Optical fibre long-period grating sensors: Characteristics and application. Meas. Sci. Technol. 2003, 14, r49-r61.

13. Elosua, C.; Matias, I.R.; Bariain, C.; Arregui, F.J. Volatile organic compound optical fiber sensors: A review. Sensors 2006, 6, 1440-1465.

14. Xu, X.; Wang, H.; Liu, J.; Yan, H. The applications of zeolitic imidazolate framework-8 in electrical energy storage devices: A review. J. Mater. Sci. Mater. Electron. 2017, 28, 7532-7543.

15. Lu, G.; Hupp, J.T. Metal-organic frameworks as sensors: A ZIF-8 based fabry-pérot device as a selective sensor for chemical vapors and gases. J. Am. Chem. Soc. 2010, 132, 7832-7833,.

16. Hromadka, J.; Tokay, B.; James, S.; Tatam, R.P.; Korposh, S. Optical fibre long period grating gas sensor modified with metal organic framework thin films. Sens. Actuators B Chem. 2015, 221, 891-899.

17. Hromadka, J.; Correia, R.; Korposh, S. Fabrication of fiber optic long period gratings operating at the phase matching turning point using an amplitude mask. In Proceedings of the SPIE-The International Society for Optical Engineering, Limerick, Ireland, 31 May 2016; Volume 9916.

18. Hromadka, J.; Tokay, B.; Korposh, S.; James, S.; Tatam, R.P. Detection of volatile organic compounds using optical fibre long period grating modified with metal organic framework thin films. In Proceedings of the SPIE-The International Society for Optical Engineering, Curitiba, Brazil, 28 September 2015; Volume 9634.

(C) 2017 by the authors. Licensee MDPI, Basel, Switzerland. This article is an open access article distributed under the terms and conditions of the Creative Commons Attribution (CC BY) license (http://creativecommons.org/licenses/by/4.0/). 